\title{
Neutron stars with the Bose-Einstein condensation of antikaons as MIT Bags
}

\author{
C. Y. Ryu, ${ }^{1, *}$ C. H. Hyun, ${ }^{2}$ and S. W. Hong ${ }^{1}$ \\ ${ }^{1}$ Department of Physics and Institute of Basic Science, \\ Sungkyunkwan University, Suwon 440-746 \\ ${ }^{2}$ Department of Physics Education, Daegu University, Gyeongsan 712-714
}

(Dated: December 26, 2008)

\begin{abstract}
We investigate the properties of an antikaon in medium, regarding it as a MIT bag. We first construct the MIT bag model for a kaon with $\sigma^{*}$ and $\phi$ in order to describe the interaction of s-quarks in hyperonic matter in the framework of the modified quark-meson coupling model. The coupling constant $g_{\sigma}^{B_{K}}$ in density dependent bag constant $B(\sigma)$ is treated as a free parameter to reproduce the optical potential of a kaon in symmetric matter, and all other couplings are determined by $\mathrm{SU}(6)$ symmetry and the quark counting rule. With various values of the kaon potential, we calculate the effective mass of a kaon in medium to compare it with that of a pointlike kaon. We then calculate the population of octet baryons, leptons and $K^{-}$, and the equation of state for the neutron star matter. The results show that kaon condensation in hyperonic matter is sensitive to the s-quark interaction and how to treat the kaon. The mass and radius of a neutron star are obtained by solving Tolmann-Oppenheimer-Volkoff equation.
\end{abstract}

*Electronic address: cyryu@skku.edu 


\section{INTRODUCTION}

The properties of an antikaon in medium are attracting much interests recently. Since a theoretical calculation [1] predicted deeply bound $K^{-}$states in $K^{-} p p, K^{-} p p n$ and $K^{-} p n n$ systems, many studies have been reported in both theory and experiment. The KEK group [2] reported the observation of $S^{0}(3115)$ and $S^{+}(3140)$ states, interpreting them as deeply bound states of $K^{-}$, though in a more recent experiment [3] with a better statistics the existence of $S^{0}$ state could not be confirmed. FINUDA group also reported the observation of a deeply bound state for $K^{-} p p$ with binding energy $B=115 \pm 9 \mathrm{MeV}$ and width $\Gamma=67 \pm 16$ $\mathrm{MeV}$ [4]. On the other hand, the authors in Refs. [5, 6] claimed that the observed state could be explained in terms of the final state interaction of produced $\Lambda p$ pairs. Therefore, further studies are needed both theoretically and experimentally to confirm such states. If a deeply bound kaonic state exists indeed, it can be formed by very strong attraction between nucleons and an antikaon, which may lead to kaon condensation in dense nuclear matter.

Possible existence of kaon condensation in dense matter was proposed about two decades ago [7]. The subject has been studied with various models such as chiral perturbation theory [7-10], relativistic mean field models like quantum-hadrodynamics (QHD) [11-13] and quarkmeson coupling (QMC) model [14, 15]. In relativistic mean field approach, the interaction among baryons is described by scalar $(\sigma)$ and vector $(\omega)$ meson fields mediating attractive and repulsive forces, respectively. In the mean field approach, due to the antiparticle nature, an antikaon feels strong attraction by both scalar and vector meson fields, which determines the optical potential of an antikaon. The kaon condensation in medium is known to be sensitive to the value of the optical potential. When we consider an antikaon in medium, it can be treated as a point-like particle in view of its property as a pseudo-Goldstone boson. However, applying the OZI rule, which says the s-quark does not interact with $\sigma$ and $\omega$ mesons, the optical potential of an antikaon depends only on the interaction of $\bar{u}$, which can feel strong attraction due to the exchange of $\sigma$ and $\omega$ mesons. Therefore it is worthwhile to treat an antikaon as a MIT bag and compare the values of physical observables with those obtained from a point-like kaon.

The QMC model originally developed by Guichon [16] assumes that quarks inside baryon bags interact with each other through the exchange of $\sigma, \omega$ and $\rho$ meson fields. The model has been further developed by several authors [17-21]. An important revision of the model 
was made in Ref. [18] by introducing a density dependent bag constant to simulate partial deconfinement of quarks at high densities and to get the meson field strengths as predicted by relativistic phenomenology. This model was called the modified QMC (MQMC) model. Then a model for a kaon as a MIT bag was proposed in Ref. [20] in the framework of QMC model in asymmetric matter, in which the interaction between nucleons and a kaon was mediated by $\sigma, \omega$ and $\rho$ meson fields. The kaon condensation in hyperonic nuclear matter was then studied in Ref. [14] with the QMC model, including $\sigma, \omega$ and $\rho$ meson fields, but the interaction between s-quarks and the density dependence of the bag constant were not considered.

In this work, we extend the previous models [14, 20] to further include the interaction of s-quarks through the exchange of $\sigma^{*}$ and $\phi$ meson fields, and assume the density dependence of the bag constants. We then study the Bose-Einstein condensation of $K^{-}$in the neutron star with the density dependent bag constant for a kaon bag as in Ref. [21] in the framework of the MQMC model. The coupling constant $g_{\sigma}^{\prime B_{K}}$ for the density dependent bag constant of a kaon is regarded as a free parameter to be determined from the kaon optical potential, which we choose as $U_{K}=-120,-140,-160 \mathrm{MeV}$. Our results show that the EoS with kaon condensation differs significantly depending on whether we treat a kaon as a point-like particle or a MIT bag. Because of the repulsion due to the s-quark interaction at high densities, the equation of states (EoS) from the QHD and QMC models without s-quark interactions are softer than those from the models including the s-quark interaction. We find that the interaction between s-quarks, which was not included in Ref. [14] can make the EoS quite different.

In Section II, we present our model, extending the previous QMC model [20] for a kaon to include the density dependence of the bag constant (i.e., MQMC model) and the interaction between the s-quarks in hyperonic nuclear matter. By treating a kaon as a MIT bag we calculate the equations of motion for mesons with kaon condensation. Energy density and pressure of the neutron star matter with kaon bags are obtained.

In Section III, we show the numerical results for the EoS and population profile of neutron star matter with hyperons at densities up to ten times normal density. Using the calculated EoS, we obtain the mass-radius relation of neutron star with hyperons and kaons. Summary follows in Section IV. 


\section{MODELS}

\section{A. Kaon and antikaon as MIT bags in hyperonic matter}

In the (M)QMC model, baryons are regarded as MIT bags and the interaction between the baryons is mediated by the exchange of meson fields among quarks inside the baryon bags. As mentioned in the Introduction, we treat a kaon as a MIT bag in this work. Thus we use the same framework for both kaons and baryons, treating all virtual meson fields as point-like particles. In Ref. [20] a kaon and an antikaon as MIT bags in nuclear matter were assumed to interact with nucleons through the interaction between the $u$ - and $d$-quarks and $\sigma, \omega$ and $\rho$ meson fields, while s-quarks were assumed to be non-interacting with any particles. Here, we extend the model of Ref. [20] to include the interaction between the s-quarks inside the kaon and baryon bags through $\sigma^{*}$ and $\phi$ meson fields. To show explicitly how kaon bags interact with five meson fields, we start with the Dirac equations for quarks and antiquarks in mean field approximation

$$
\begin{gathered}
{\left[i \gamma \cdot \partial-\left(m_{u}-g_{\sigma}^{q} \sigma\right) \mp \gamma^{0}\left(g_{\omega}^{q} \omega_{0}+\frac{1}{2} g_{\rho}^{q} \rho_{03}\right)\right]\left(\begin{array}{l}
u \\
\bar{u}
\end{array}\right)=0} \\
{\left[i \gamma \cdot \partial-\left(m_{d}-g_{\sigma}^{q} \sigma\right) \mp \gamma^{0}\left(g_{\omega}^{q} \omega_{0}-\frac{1}{2} g_{\rho}^{q} \rho_{03}\right)\right]\left(\begin{array}{l}
d \\
\bar{d}
\end{array}\right)=0} \\
{\left[i \gamma \cdot \partial-\left(m_{s}-g_{\sigma^{*}}^{q} \sigma^{*}\right) \mp \gamma^{0} g_{\phi}^{q} \phi_{0}\right]\left(\begin{array}{c}
s \\
\bar{s}
\end{array}\right)=0,}
\end{gathered}
$$

where $\omega_{0}$ and $\phi_{0}$ are, respectively, the time components of $\omega$ and $\phi$ meson fields and $\rho_{03}$ is the z-component of the time-component of $\rho$ meson field. For $\mathrm{u}$ and $\mathrm{d}$-quarks we use $m_{u}=m_{\bar{u}}=m_{d}=m_{\bar{d}}=0$, while for s and $\bar{s}$ quarks, $m_{s}=m_{\bar{s}}=150 \mathrm{MeV}$ is used. In the assumption that all the quarks are in the ground state, we can use the following normalized, static solution for the quarks in a kaon bag,

$$
\psi_{i}(\vec{r}, t)=N_{i} e^{i \epsilon_{i} t / R_{K}} \phi_{i}(\vec{r}), \quad i=u, \bar{u}, d, \bar{d}, s, \bar{s}
$$

where $N_{i}, \phi_{i}(\vec{r})$ and $R_{K}$ are the normalization factor, the spatial part of the wave function and the bag radius of a kaon bag, respectively. The eigenenergies of quarks in units of $1 / R_{K}$ can be obtained as

$$
\left(\begin{array}{c}
\epsilon_{u} \\
\epsilon_{\bar{u}}
\end{array}\right)=\Omega_{u} \pm R_{K}\left(g_{\omega}^{q} \omega_{0}+\frac{1}{2} g_{\rho}^{q} \rho_{03}\right)
$$




$$
\begin{aligned}
& \left(\begin{array}{c}
\epsilon_{d} \\
\epsilon_{\bar{d}}
\end{array}\right)=\Omega_{d} \pm R_{K}\left(g_{\omega}^{q} \omega_{0}-\frac{1}{2} g_{\rho}^{q} \rho_{03}\right) \\
& \left(\begin{array}{c}
\epsilon_{s} \\
\epsilon_{\bar{s}}
\end{array}\right)=\Omega_{s} \pm R_{K} g_{\phi}^{q} \phi_{0}
\end{aligned}
$$

where $\Omega_{q}=\sqrt{x_{q}^{2}+\left(R_{K} m_{q}^{*}\right)^{2}}$ with $m_{q}^{*}=m_{q}-g_{\sigma}^{q} \sigma$ for $q=u, \bar{u}, d, \bar{d}$ and for $q=s$ and $\bar{s}$ $\Omega_{s}=\sqrt{x_{s}^{2}+\left(R_{K} m_{s}^{*}\right)^{2}}$ with $m_{s}^{*}=m_{s}-g_{\sigma^{*}}^{q} \sigma^{*}$. The energies of a kaon and an antikaon can be obtained through the sum of the energies of their quark and antiquark components as

$$
\begin{aligned}
& \left(\begin{array}{c}
\omega_{K^{+}} \\
\omega_{K^{-}}
\end{array}\right)=m_{K}^{*} \pm\left(g_{\omega}^{q} \omega_{0}-g_{\phi}^{q} \phi_{0}+\frac{1}{2} g_{\rho}^{q} \rho_{03}\right) \\
& \left(\begin{array}{c}
\omega_{K^{0}} \\
\omega_{\bar{K}^{0}}
\end{array}\right)=m_{K}^{*} \pm\left(g_{\omega}^{q} \omega_{0}-g_{\phi}^{q} \phi_{0}-\frac{1}{2} g_{\rho}^{q} \rho_{03}\right)
\end{aligned}
$$

where the effective mass of a kaon $m_{K}^{*}$ is calculated by eliminating the spurious motion of quarks in a bag from the bag energy [17, 21],

$$
m_{K}^{*}=\sqrt{E_{K}^{2}-\sum_{q}\left(\frac{x_{q}}{R_{K}}\right)^{2}} .
$$

Here the bag energy is given as

$$
E_{K}=\frac{\Omega_{u}+\Omega_{s}-Z_{K}}{R_{K}}+\frac{4}{3} \pi R_{K}^{3} B_{K}
$$

with the bag constant $B_{K}$ and a phenomenological constant $Z_{K}$, which are determined by the minimum condition

$$
\left.\frac{\partial m_{K}}{\partial R}\right|_{R=R_{K}}=0
$$

to produce the free mass of a kaon in vacuum with $R_{K}=0.4 \mathrm{fm}$. In eq. (8), one can see both $\sigma$ and $\omega$ mesons give the attraction to an antikaon, while the $\phi$ meson fields give the repulsion. In addition, because the value of $\rho_{03}$ is usually negative in asymmetric matter, both $\phi$ and $\rho$ meson fields play a role to prohibit the kaon condensation in the neutron star matter.

In the MQMC model, the baryon bag constant $B$ in medium depends on $\sigma$ and $\sigma^{*}$. For the kaon bag also we use the density dependent bag constant given by

$$
B_{K}\left(\sigma, \sigma^{*}\right)=B_{0} \exp \left[-4 g_{\sigma}^{\prime B_{K}}\left(\sigma+\sqrt{2} \sigma^{*}\right) / m_{K}\right]
$$


where $B_{0}$ and $m_{K}$ are the bag constant and the mass of a kaon in vacuum, respectively. Here the factor $\sqrt{2}$ is from the $\mathrm{SU}(6)$ symmetry. By applying a boost, we can obtain a general dispersion relation for a kaon and an antikaon with momentum $\vec{k}$. We can write a Lagrangian for a kaon and an antikaon bag interacting with meson fields as

$$
\mathcal{L}_{K}=\left(D^{\mu} K\right)^{\dagger} D_{\mu} K-m_{K}^{*}{ }^{\dagger} K^{\dagger} K
$$

where the covariant derivative $D_{\mu}=\partial_{\mu}+i g_{\omega}^{K} \omega_{\mu}-i g_{\phi}^{K} \phi_{\mu}+i g_{\rho}^{K} \frac{\tau_{3}}{2} \rho_{\mu}$. For the s-wave $(\vec{k}=0)$, we can easily check that this Lagrangian provides the dispersion relations in Eqs. (8) and (9). Different signs in front of $\omega$ and $\phi$ meson fields originate from the quark and antiquark components of a kaon.

\section{B. Kaon condensation in neutron star}

The total Lagrangian for hyperonic matter with baryon octet, five meson fields, leptons and (anti) kaons in mean field approximation is given by

$$
\mathcal{L}=\mathcal{L}_{\text {matter }}+\mathcal{L}_{K}
$$

where

$$
\begin{aligned}
\mathcal{L}_{\text {matter }} & =\sum_{B} \bar{\psi}_{B}\left[i \gamma \cdot \partial-m_{B}^{*}\left(\sigma, \sigma^{*}\right)+\gamma^{0}\left(\omega_{0}+\phi_{0}+\frac{1}{2} \tau_{3} \rho_{03}\right)\right] \psi_{B} \\
& +\frac{1}{2} m_{\omega}^{2} \omega_{0}^{2}+\frac{1}{2} m_{\phi}^{2} \phi_{0}^{2}+\frac{1}{2} m_{\rho}^{2} \rho_{03}^{2}+\sum_{l} \bar{\psi}_{l}\left(i \gamma \cdot \partial-m_{l}\right) \psi_{l}
\end{aligned}
$$

and $\mathcal{L}_{K}$ is given by Eq. (14). The effective mass of a baryon as a MIT bag $m_{B}^{*}\left(\sigma, \sigma^{*}\right)$ can be written as [17, 22]

$$
m_{B}^{*}=\sqrt{E_{B}^{2}-\sum_{q}\left(\frac{x_{q}}{R}\right)^{2}}
$$

where the bag energy of a baryon is given by

$$
E_{B}=\sum_{q} \frac{\Omega_{q}}{R}-\frac{Z_{B}}{R}+\frac{4}{3} \pi R^{3} B_{B}
$$

The bag constant $B_{B}$ and a phenomenological constant $Z_{B}$ are fitted to reproduce the free mass of each baryon at a given bag radius $R$, respectively. In MQMC model $B_{B}$ depends 
on the matter density and can be written as

$$
B_{B}\left(\sigma, \sigma^{*}\right)=B_{B 0} \exp \left\{-4 g_{\sigma}^{\prime B}\left(\sum_{q=u, d} n_{q} \sigma+\left(3-\sum_{q=u, d} n_{q}\right) \sqrt{2} \sigma^{*}\right) / m_{B}\right\},
$$

where $m_{B}$ is the bare mass of the baryon $B$ and the factor $\sqrt{2}$ is from the $\mathrm{SU}(6)$ symmetry.

When considering the s-wave $K^{-}$condensation, the equations for five meson fields are obtained as

$$
\begin{gathered}
m_{\sigma}^{2} \sigma=\sum_{B} g_{\sigma B} C_{B}(\sigma) \frac{2 J_{B}+1}{2 \pi^{2}} \int_{0}^{k_{B}} \frac{m_{B}^{*}}{\left[k^{2}+m_{B}^{* 2}\right]^{1 / 2}} k^{2} d k+g_{\sigma K} C_{K}(\sigma) \rho_{K}, \\
m_{\sigma^{*}}^{2} \sigma^{*}=\sum_{B} g_{\sigma^{*} B} C_{B}\left(\sigma^{*}\right) \frac{2 J_{B}+1}{2 \pi^{2}} \int_{0}^{k_{B}} \frac{m_{B}^{*}}{\left[k^{2}+m_{B}^{* 2}\right]^{1 / 2}} k^{2} d k+g_{\sigma^{*} K} C_{K}\left(\sigma^{*}\right) \rho_{K}, \\
m_{\omega}^{2} \omega_{0}=\sum_{B} g_{\omega B}\left(2 J_{B}+1\right) k_{B}^{3} /\left(6 \pi^{2}\right)-g_{\omega K} \rho_{K}, \\
m_{\phi}^{2} \phi_{0}=\sum_{B} g_{\phi B}\left(2 J_{B}+1\right) k_{B}^{3} /\left(6 \pi^{2}\right)+g_{\phi K} \rho_{K}, \\
m_{\rho}^{2} \rho_{03}=\sum_{B} g_{\rho B} I_{3 B}\left(2 J_{B}+1\right) k_{B}^{3} /\left(6 \pi^{2}\right)-g_{\rho K} I_{3 K} \rho_{K} .
\end{gathered}
$$

In the above equations $J_{B}$ and $I_{3 B}$ are the spin and the isospin projection and $k_{B}$ is the Fermi momentum of the baryon species $B$. The factors in Eqs. (21) and (22), $C_{B}(\sigma)$, $C_{B}\left(\sigma^{*}\right), C_{K}(\sigma)$ and $C_{K}\left(\sigma^{*}\right)$ are, respectively, given by

$$
\begin{aligned}
g_{\sigma B} C_{B}(\sigma) & =-\frac{\partial m_{B}^{*}}{\partial \sigma}, \\
g_{\sigma^{*} B} C_{B}\left(\sigma^{*}\right) & =-\frac{\partial m_{B}^{*}}{\partial \sigma^{*}}, \\
g_{\sigma K} C_{K}(\sigma) & =-\frac{\partial m_{K}^{*}}{\partial \sigma}, \\
g_{\sigma^{*} K} C_{K}\left(\sigma^{*}\right) & =-\frac{\partial m_{K}^{*}}{\partial \sigma^{*}} .
\end{aligned}
$$

Detailed expressions for Eqs. (26) and (27) are given in Refs. [18, 22]. $g_{\sigma K} C_{K}(\sigma)$ and $g_{\sigma^{*} K} C_{K}\left(\sigma^{*}\right)$ for a point-like kaon are nothing but $g_{\sigma K}$ and $g_{\sigma^{*} K}$, respectively, but for a kaon as a MIT bag they need to be calculated self-consistently. The detailed expressions can be written similarly as in [18, 22]. 
Let us now apply this model for hyperonic matter with kaon bags to a neutron star matter. Neutron star matter is characterized by three conditions; baryon number conservation, charge neutrality and chemical equilibrium. Baryons can be produced when the chemical equilibrium conditions are satisfied

$$
\begin{gathered}
\mu_{n}=\mu_{\Lambda}=\mu_{\Sigma^{0}}=\mu_{\Xi^{0}}, \\
\mu_{n}+\mu_{e}=\mu_{\Sigma^{-}}=\mu_{\Xi^{-}}, \\
\mu_{n}-\mu_{e}=\mu_{p}=\mu_{\Sigma^{+}}
\end{gathered}
$$

where the chemical potential of a baryon is given by

$$
\mu_{B}=\sqrt{k_{B}^{2}+m_{B}^{* 2}\left(\sigma, \sigma^{*}\right)}+g_{\omega B} \omega_{0}+g_{\phi B} \phi_{0}+g_{\rho B} I_{3 B} \rho_{03}
$$

and that of a lepton is simply written as

$$
\mu_{l}=\sqrt{k_{l}^{2}+m_{l}^{2}}
$$

Also, the density of a muon is determined by $\mu_{e}=\mu_{\mu}$. When kaon condensation takes place, electrons are replaced by $K^{-}$so that $n \rightarrow p+K^{-}$. Therefore, kaons are produced when the condition $\mu_{n}-\mu_{p}=\mu_{K}$ is met, $\mu_{K}$ being the chemical potential of a kaon equal to the kaon energy in Eq. (8) for S-wave condensation. Also, the charge neutrality gives us the condition

$$
\sum_{B} q_{B} \rho_{B}-\rho_{K}-\rho_{e}-\rho_{\mu}=0
$$

where $q_{B}$ is the charge and $\rho_{B}$ is the number density of baryon species $B$.

The energy density of the matter gets contributions from all the particles,

$$
\begin{aligned}
\varepsilon= & \frac{1}{2} m_{\sigma}^{2} \sigma^{2}+\frac{1}{2} m_{\sigma^{*}}^{2} \sigma^{* 2}+\frac{1}{2} m_{\omega}^{2} \omega_{0}^{2}+\frac{1}{2} m_{\phi}^{2} \phi_{0}^{2}+\frac{1}{2} m_{\rho}^{2} \rho_{03}^{2} \\
& +\sum_{B} \frac{2 J_{B}+1}{2 \pi^{2}} \int_{0}^{k_{B}}\left[k^{2}+m_{B}^{* 2}\right]^{1 / 2} k^{2} d k+\sum_{l} \frac{1}{\pi^{2}} \int_{0}^{k_{l}}\left[k^{2}+m_{l}^{2}\right]^{1 / 2} k^{2} d k \\
& +m_{K}^{*} \rho_{K},
\end{aligned}
$$

but because there is no contribution to pressure from a kaon in its s-wave the pressure is given by

$$
\begin{aligned}
P= & -\frac{1}{2} m_{\sigma}^{2} \sigma^{2}-\frac{1}{2} m_{\sigma^{*}}^{2} \sigma^{* 2}+\frac{1}{2} m_{\omega}^{2} \omega_{0}^{2}+\frac{1}{2} m_{\phi}^{2} \phi_{0}^{2}+\frac{1}{2} m_{\rho}^{2} \rho_{03}^{2} \\
& +\frac{1}{3} \sum_{B} \frac{2 J_{B}+1}{2 \pi^{2}} \int_{0}^{k_{B}} \frac{k^{4} d k}{\left[k^{2}+m_{B}^{* 2}\right]^{1 / 2}}+\frac{1}{3} \sum_{l} \frac{1}{\pi^{2}} \int_{0}^{k_{l}} \frac{k^{4} d k}{\left[k^{2}+m_{l}^{2}\right]^{1 / 2}} .
\end{aligned}
$$




\begin{tabular}{|c|c|c|c|c|c|c|}
\hline$g_{\sigma}^{q}$ & $g_{\omega}^{q}$ & $g_{\sigma}^{\prime}$ & $g_{\rho}^{q}$ & $m_{N}^{*} / m_{N}$ & $K(\mathrm{MeV})$ & $a_{\text {sym }}(\mathrm{MeV})$ \\
\hline 1.0 & 2.71 & 2.27 & 7.89 & 0.78 & 285.5 & 32.5 \\
\hline
\end{tabular}

TABLE I: The coupling constants for $(u, d)$-quarks and $(\sigma, \omega, \rho)$-mesons in the MQMC model to reproduce the binding energy $B / A=16 \mathrm{MeV}$ at the saturation density $\rho_{0}=0.17 \mathrm{fm}^{-3}$ and symmetry energy $a_{\text {sym }}=32.5 \mathrm{MeV} \cdot m_{N}^{*} / m_{N}$ and $K$ are the ratio of the effective mass to the free mass of the nucleon and the compression modulus at the saturation density, respectively [15].

\section{Parameters}

For the bag constant $B$ and $Z_{B}$ of baryons, we use the parameters given in Ref. [15] which are fitted to reproduce the free masses of baryons. The values of $B_{K}$ and $Z_{K}$ for a kaon are determined to fit the free mass of a kaon with $R_{K}=0.4 \mathrm{fm}, m_{u(d)}=0 \mathrm{MeV}$ and $m_{s}=150 \mathrm{MeV}$. The values are $B_{K}^{1 / 4}=170.752 \mathrm{MeV}$ and $Z_{K}=1.152$. The coupling constants between $\mathrm{u}(\mathrm{d})$ quarks and $\sigma, \omega$ and $\rho$ mesons are determined to reproduce nuclear matter properties at the saturation density $\left(\rho_{0}=0.17 \mathrm{fm}^{-3}\right)$. The coupling parameters determined for $g_{\sigma}^{u}=1.0$ are shown in Table [ taken from Ref. [15]. The couplings between the s-quark and $\sigma^{*}$ and $\phi$ are given by the $\mathrm{SU}(6)$ symmetry. In this work, we assume that the s-quark does not interact with $\sigma, \omega$ and $\rho$ mesons.

The coupling constants for the interaction between the octet baryons and mesons can be determined by using the coupling constants in Table \and the quark counting rule

$$
\begin{aligned}
g_{\omega}^{q} & =\frac{1}{3} g_{\omega N}=\frac{1}{2} g_{\omega \Lambda}=\frac{1}{2} g_{\omega \Sigma}=g_{\omega \Xi}, \\
g_{\rho}^{q} & =g_{\rho N}=g_{\rho \Sigma}=g_{\rho \Xi}, \quad g_{\rho \Lambda}=0 \\
g_{\phi}^{s} & =g_{\phi \Lambda}=g_{\phi \Sigma}=\frac{1}{2} g_{\phi \Xi}
\end{aligned}
$$

where $g_{\phi}^{s}=\sqrt{2} g_{\omega}^{u, d}$ from the $\mathrm{SU}(6)$ symmetry.

For coupling constants between the kaon and mesons, $g_{\omega K}$ and $g_{\rho K}$ can be obtained by the quark counting rule $\left(g_{\omega K}=g_{\omega}^{q}\right.$ and $\left.g_{\rho K}=g_{\rho}^{q}\right), g_{\sigma^{*} K}$ can be fixed from $f_{0}(980)$ decay, and $g_{\phi K}$ from the $\mathrm{SU}(6)$ relation $\sqrt{2} g_{\phi K}=g_{\pi \pi \rho}$. Then we get $g_{\sigma^{*} K}=2.65$ and $g_{\phi K}=4.27$. The value of $g_{\sigma}^{\prime B_{K}}$ is associated with the depths of the antikaon potential in medium through the relation

$$
U_{K}=-\left(m_{K}-m_{K}^{*}\right)-g_{\omega K} \omega_{0}
$$




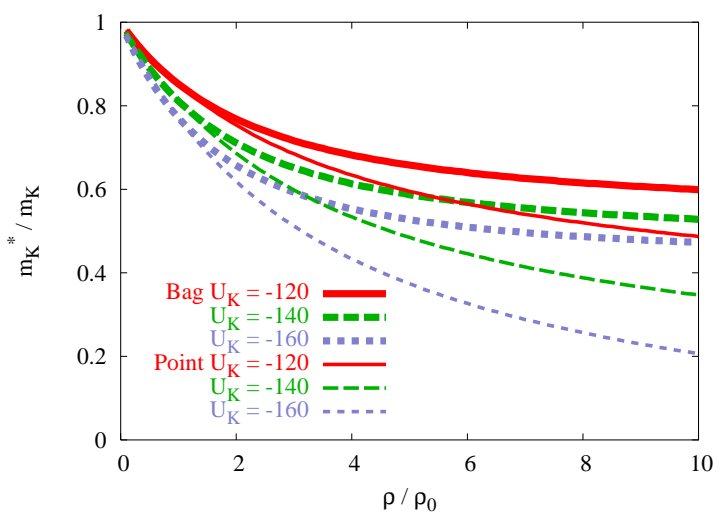

FIG. 1: The effective mass of a kaon in symmetric nuclear matter is plotted for both MIT bag kaons (thick curves) and point-particle kaons (thin curves).

and Eqs. (10) and (11). With $U_{K}=-120,-140$ and $-160 \mathrm{MeV}$ and $g_{\sigma}^{q}=1$, we obtain $g_{\sigma}^{B_{K}}$ as $3.114,4.317$ and 5.615 , respectively.

\section{RESULTS}

The effective mass of a kaon bag in the MQMC model in a symmetric nuclear matter is calculated with Eq. (10) and compared with that of a point-like kaon in Fig. 1. For a pointlike kaon, the effective mass is simply given by $m_{K}^{*}=m_{K}-g_{\sigma K} \sigma$. For a point-like kaon, $g_{\sigma K}$ is fixed to reproduce the given value of the kaon optical potential $U_{K}=-g_{\sigma K} \sigma-g_{\omega K} \omega_{0}$. Up to $\rho \sim 2 \rho_{0}$, the effective mass decreases similarly for both point particle and MIT bag kaons. For $\rho>2 \rho_{0}$, the behaviors are contrasting. The reduction of the mass of a kaon bag saturates more or less at high densities, while the mass of a point-like kaon keeps decreasing.

In Figs. 2 and 3, fractional population of particles and the EoS of neutron star matter are shown for the matter with nucleons, leptons and kaons $\left(n p K^{-}\right.$; left column), for the matter with octet baryons, leptons and kaons without s-quark interaction (middle column), and for the matter with octet baryons, leptons and kaons with s-quark interaction (right column). Each row in Fig. 2 corresponds to $U_{K}=-120,-140$, and $-160 \mathrm{MeV}$, respectively, from the top.

In the case of nuclear matter with $n p K^{-}$, the onset densities of kaon condensation are quite low as 3.6, 3.2 and 2.9 times $\rho_{0}$ for $U_{K}=-120,-140$ and $-160 \mathrm{MeV}$, respectively. Even the largest onset density $3.6 \rho_{0}$ is similar to the mean density of a typical neutron star, 

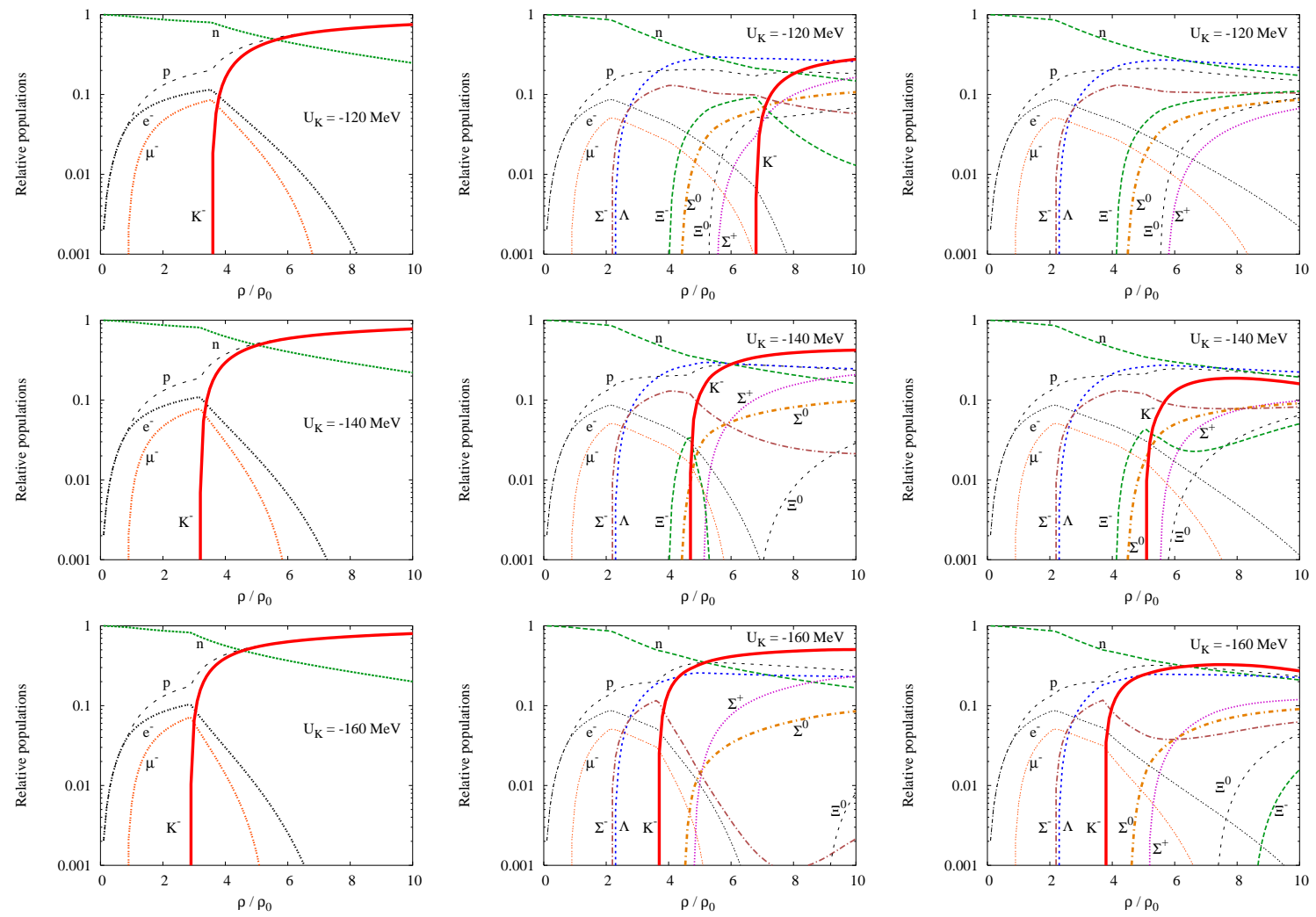

FIG. 2: The relative populations of particles in nuclear matter (left column), hyperonic matter without s-quark interactions (middle column), and hyperonic matter with s-quark interactions (right column) for the kaon potential $U_{K}=-120,-140,-160 \mathrm{MeV}$, respectively.
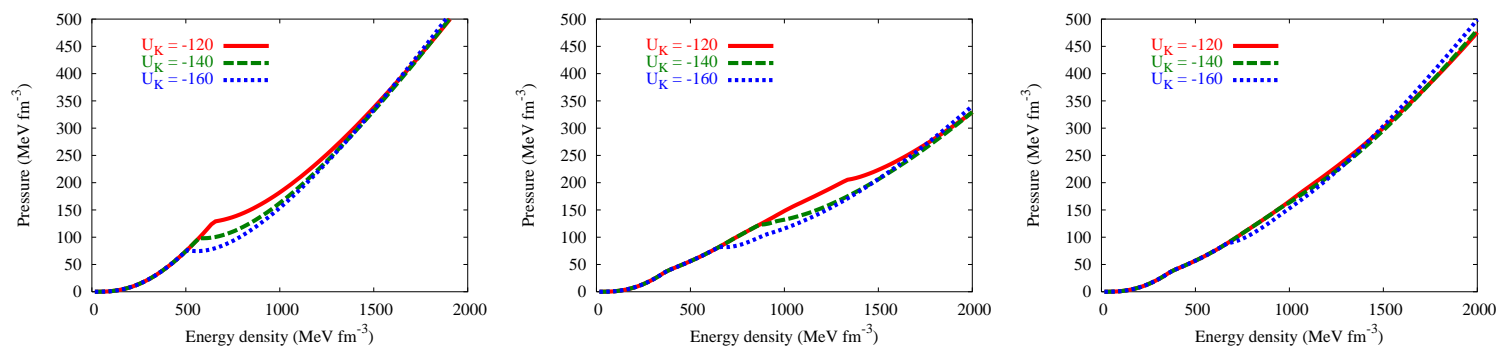

FIG. 3: The equation of state for nuclear matter with only n, p and K (left), hyperonic matter (n, p, Y, K) without s-quark interactions (middle), and hyperonic matter with s-quark interactions (right), respectively.

mass $\sim 2.0 M_{\odot}$ and radius $R \sim 10 \mathrm{~km}$. Thus, if pure nucleonic phase persists to a certain depth of the neutron star, kaon condensation is likely to be the state of matter inside the neutron star. At high densities, population of $K^{-}$is dominant, making the neutron star a 
kaonic matter.

The EoS for $n p K^{-}$matter is shown in the left panel of Fig. 3. It is worthwhile to compare our result with that obtained with point-like kaon in the QHD model [12]. In Ref. [12] the phase transition is treated as first-order and a very soft EoS is obtained. On the other hand, because the softening of EoS due to the kaon condensation is not so substantial in our model, the phase transition from normal nuclear phase to kaonic phase depends on how a kaon is treated in medium as well as kaon optical potetial. However, once the kaon condensation sets in, the particle composition becomes very similar at high densities regardless of the $U_{K}$ value, and it makes the EoS also similar at high densities.

With hyperon degrees of freedom but without s-quark interactions ( $n p Y K^{-}$matter), we obtain the composition of the neutron star matter shown in the middle column of Fig. 2 , Since antikaon has a negative charge and becomes very dominant once it is created, all the negatively charged hyperons disappear soon after the kaon condensation. Condensation onset densities are 6.8, 4.5 and 3.8 times $\rho_{0}$ for $U_{K}=-120,-140$ and $-160 \mathrm{MeV}$, respectively. Dependence of kaon condensation on the value of $U_{K}$ is stronger than the $n p K^{-}$ matter case. The reason for this strong dependence may be partially attributed to the existence of the hyperons before the kaon condensation. As density increases, Fermi momentum of the neutrons reaches to a value that satisfies the chemical equilibrium for light hyperons such as $\Lambda$ and $\Sigma^{-}$. The creation of the $\Lambda$ and $\Sigma^{-}$hyperons reduces the rate of increase of the neutron Fermi momentum as a function of density, but the proton Fermi momentum is hardly affected by the hyperons. Then since $\mu_{n}-\mu_{p}$ becomes smaller than that without the hyperons, kaon creation condition $\mu_{K}=\mu_{n}-\mu_{p}$ becomes more sensitive to the behavior of the kaon chemical potential.

The EoS for $n p Y K^{-}$matter, shown in the middle panel of Fig. 3 is much softer than that for $n p K^{-}$matter because of the creation of the hyperon. The softening of the EoS due to kaons on top of the creation of hyperons is less significant. For instance, the derivative of the $\operatorname{EoS} d P / d \varepsilon$ for $n p K^{-}$matter with $U_{K}=-160 \mathrm{MeV}$ abruptly becomes close to zero at the density where the kaon condensation begins, and the gap between the two EoS's for $U_{K}=-120 \mathrm{MeV}$ and $-160 \mathrm{MeV}$ is substantial. On the other hand, the derivative $d P / d \varepsilon$ for $n p Y K^{-}$matter undergoes only a slight decrease at the density of kaon creation, and so it remains similar to the value without the kaon condensation. Kaon condensation plays a significant role in the EoS if hyperons are not included, but with the inclusion of hyperons 
the kaon condensation becomes less significant.

Now, if we switch on the interaction of s-quarks with $\sigma^{*}$ and $\phi$ mesons $\left(n p Y K^{-} \phi\right)$ on top of including the hyperon degrees of freedom, we get the particle composition in the right column of Fig. 2. When $U_{K}=-120 \mathrm{MeV}$, kaon condensation does not take place at all up to ten times the saturation density. For $U_{K}=-140$ and $-160 \mathrm{MeV}$, kaon condensation sets in at 5.1 and 3.8 times $\rho_{0}$, respectively. The onset density of the kaon condensation is more sensitive to the value of $U_{K}$ than in the $n p Y K^{-}$case. In addition, the population of the kaon is significantly reduced from that in the case of $n p Y K^{-}$matter for all the $U_{K}$ values. The population of s-quarks increases at higher densities due to large production of hyperons and kaons. Thus the values of $\sigma^{*}$ - and $\phi$ - fields increase because strangeness is the source of these meson fields, but at high densities, $\phi$ meson fields increase more rapidly than $\sigma^{*}[15]$. Therefore, the repulsion due to $\phi$ mesons inside the hyperon and kaon bags increase as the population of hyperons and kaons increase. This repulsion suppresses the kaon condensation. An interesting thing is that our results show the repulsion affects the onset of kaon condensation very much, but the effect is small for the hyperons. This can be understood by considering the attraction and repulsion coming from $\sigma$ and $\omega$ fields, respectively. The net mean field potential of a baryon is a result of the cancelation between a huge attraction due to $\sigma$ mesons and a huge repulsion due to $\omega$ mesons. With the s-quark interaction, the net result after the cancelation between $\sigma^{*}$ and $\phi$ does not affect much the population of baryons. For instance, the onset density of $\Xi^{-}$with s-quark interaction changes only slightly from that without the s-quark interaction. However, in case of the antikaon, both $\sigma$ and $\omega$ mesons cause only the attraction between baryons and antikaon. As a result, the effect of repulsion due to s-quark interaction becomes significant, and in particular, in case of relatively small potential $\left(U_{K}=-120 \mathrm{MeV}\right)$, it strongly suppresses kaon condensation. On the contrary, in case of $U_{K}=-160 \mathrm{MeV}$, the effect of s-quarks is relatively small to kaon condensation because of the reason mentioned above.

We compare the present result with that of a point-like kaon in the framework of MQMC [15], where nuclear saturation properties are exactly the same as those in this work. If there is any difference, it has to come from how one treats the kaon; a bag or a point particle. First, the onset density of the kaon condensation differs significantly. For the point-like kaon, we have the onset density $5.9,3.8$ and 3.0 times $\rho_{0}$ for $U_{K}=-120,-140$, and $-160 \mathrm{MeV}$, respectively. Second, the population of the kaon is very different for the point-like and the 


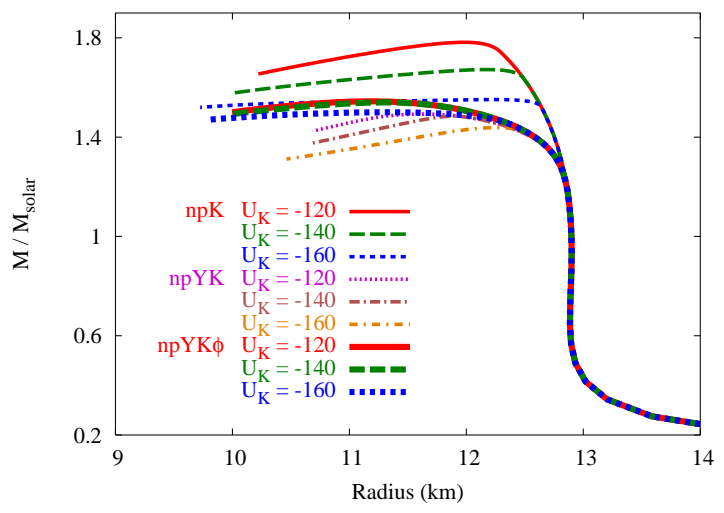

FIG. 4: The relation of mass and radius of neutron star for $n p K^{-}, n p Y K^{-}$and $n p Y K^{-} \phi$.

bag kaon. For the point-like kaon, the population of the kaon is even more dominating than that of the bag kaon without s-quark interactions. Since the background baryonic matter of the kaon condensation is built on the same ground with the same saturation properties, the same models and parameters for baryons and their interactions, the dramatic difference in the kaon condensation densities and the kaon population indicates substantial dependency on the way of how to treat the kaon; a point particle or a bag. On the other hand, we have observed from the result of the $n p Y K^{-}$case that if hyperons are included, a softening of the EoS is predominantly driven by hyperons, and the role of kaons is less significant. The EoS shown in the right panel of Fig. 3 is consistent with this observation. The EoS with strange-meson exchange is stiffer than that without it, which may be ascribed to the extra repulsion due to $\phi$ meson mean field. The existence of the hyperons, on the other hand, reduces the pressure substantially, and consequently the EoS for the $n p Y K^{-} \phi$ matter lies in between $n p K^{-}$and $n p Y K^{-}$cases.

Finally, we calculate the mass-radius relation of neutron stars by solving TolmanOppenheimer-Volkoff equation. The results are plotted in Fig. 4. The maximum mass of the star for the $n p K^{-}$case is about $1.78,1.67$ and 1.55 times the solar mass $M_{\odot}$ for $U_{K}=-120,-140$ and $-160 \mathrm{MeV}$, respectively, and the corresponding radius is about $12 \sim 13 \mathrm{~km}$. Compared with the results in Ref. [12], our results yield the stars with larger mass and radius. In particular, in Ref. [12] the radius of the kaonic star is obtained to be very small (about $8 \mathrm{~km}$ ) when kaon condensation takes place with $U_{K}=-140 \mathrm{MeV}$. Our results show that the mass and the radius of the star depend much on the model, especially, on how to treat a kaon in medium. The maximum masses of the star for the $n p Y K^{-}$matter 
are about $1.49,1.48$ and 1.44 times $M_{\odot}$ for $U_{K}=-120,-140,-160 \mathrm{MeV}$, respectively. This model give us a lighter star, which tells us that the effect of hyperons is very significant. For the neutron star with $n p Y K^{-} \phi$, the maximum mass becomes about 1.54, 1.54 and $1.50 M_{\odot}$ for $U_{K}=-120,-140$ and $-160 \mathrm{MeV}$. The stiffer EoS due to the repulsion by the s-quark interaction gives us larger values of the mass for the case $n p Y K^{-} \phi$ than the case of $n p Y K^{-}$. Depending on the value of $U_{K}$, s-quark interaction can enhance the maximum mass by $\leq 7 \%$.

The population profile and the EoS of the neutron star depend not only on the $U_{K}$ value but also on whether a kaon is treated as a point particle or a bag. With a point-like kaon, the maximum mass is in the range $(1.45 \sim 1.61) M_{\odot}$ for $U_{K}=(-160 \sim-120) \mathrm{MeV}$, an uncertainty exceeding $10 \%$. With a kaon bag, on the other hand, we have the maximum mass $(1.44 \sim 1.49) M_{\odot}$ for $n p Y K^{-}$and $(1.50 \sim 1.54) M_{\odot}$ for $n p Y K^{-} \phi$, which gives about $3 \%$ uncertainty.

\section{SUMMARY}

We have considered the existence of $K^{-}$in dense nuclear matter by treating it as a MIT bag within the framework of the MQMC model. We have investigated the effective mass of $K^{-}$in symmetric nuclear matter and its effect to the properties of the neutron star. In addition to the standard $\sigma$-, $\omega$ - and $\rho$ - meson exchanges which account for the non-strange interactions, we have also considered the interaction between strange quarks by including $\sigma^{*}$ - and $\phi$ - meson exchanges. In order to delineate the relative contributions, we have made various comparisons with and without hyperons, with and without strangeness interactions, and from shallow to deep kaon optical potential. Most importantly, we have compared various results obtained from a kaon bag and a point-like kaon.

The results without hyperons $\left(n p K^{-}\right)$show that kaon condensation occurs at the density of around $3 \rho_{0}$, and the dependence on the kaon optical potential value $U_{K}$ is relatively weak. On the other hand, the neutron star mass depends on $U_{K}$ value sensitively with the value in the range $M=(1.55 \sim 1.78) M_{\odot}$ for $U_{K}=(-160 \sim-120) \mathrm{MeV}$. Comparing our $n p K^{-}$ result with the one obtained from the QHD model in which the nucleon and the kaon are assumed as point particles [12], the results are clearly contrasting. In the QHD result, kaon condensation depends sensitively on the $U_{K}$ value, the EoS in the mixed phase is much 
softer than that in this work, and consequently the maximum mass of the neutron star is 10\% lighter than our results. Qualitatively, however, QHD and MQMC have a common aspect: the effect of the kaon condensation to the matter composition, the EoS and the neutron star properties is significant and dominant when there is no hyperons.

If we include the hyperons, $\Lambda$ and $\Sigma^{-}$hyperons are created at a density of about $2.2 \rho_{0}$. The creation of the hyperons brings down the highly accumulated neutron's Fermi levels, and it causes the role of the kaon to be minor in many respects. The onset density of the kaon condensation is more sensitive to the $U_{K}$ value than that without hyperon, and the population of the kaon is much less than that for the $n p K^{-}$case. The softening of the EoS due to kaon condensation is non-negligible even with the hyperon, but it is not so significant as that for the $n p K^{-}$matter. The neutron star mass differs by about $3 \%$ depending on $U_{K}$ value. This weak dependence on $U_{K}$ value is in contrast to that for a point-like kaon, which is about $10 \%$ [15]. With the strangeness interaction, repulsion due to $\phi$-meson exchange suppresses the role of the kaon further. As a result, the EoS for the $n p Y K^{-} \phi$ matter is almost the same as that without the kaon condensation for all the $U_{K}$ values considered. The maximum mass fluctuates by about $3 \%$ depending on the $U_{K}$ value, which may be within the uncertainties due to the model dependence, input parameters such as nuclear saturation properties, nucleon-nucleon, nucleon-hyperon and hyperon-hyperon interactions, and etc. In the case of $n p Y K^{-}$and $n p Y K^{-} \phi$ matter, compared to the point-like kaon, the role of the bag kaon is quite suppressed and the difference in the mass of the neutron star is within the errors due to various uncertainties.

\section{Acknowledgments}

The work was supported by the Korea Research Foundation Grant funded by the Korean Government(MOEHRD) (KRF-2006-214-C00015) and by the Korea Science and Engineering Foundation grant funded by the Korean Government (MEST) (No. M20608520001-08B085200110).

[1] Y. Akaishi and T. Yamazaki, Phys. Rev. C 65, 044005(2002).

[2] T. Suzuki et al., Phys. Lett. B 597, 263 (2004). 
[3] M. Sato et al., Phys. Lett. B 659, 107 (2008).

[4] M. Agnello et al., Phys. Rev. Lett. 94, 212303 (2005).

[5] E. Oset and H. Toki, Phys. Rev. C 74, 015207 (2006).

[6] V. K. Magas, A. Ramos, E. Oset and H. Toki, Phys. Rev. C 74, 025206 (2006).

[7] D. B. Kaplan and A. E. Nelson, Phys. Lett. B 175, 57 (1986).

[8] G. E. Brown and M. Rho, Phys. Rev. Lett. 66, 2720 (1991).

[9] G. E. Brown, Chang-Hwan Lee, M. Rho and V. Thorsson, Nucl. Phys. A 567, 937 (1994).

[10] T. Muto, Phys. Rev. C 77, 015810 (2008).

[11] N. K. Glendenning, J. Schaffner-Bielich, Phys. Rev. Lett. 81, 4564 (1998).

[12] N. K. Glendenning, J. Schaffner-Bielich, Phys. Rev. C 60, 025803 (1999).

[13] S. Banik and D. Bandyopadhyay, Phys. Rev. C 64, 055805 (2001).

[14] D. P. Menezes, P. K. Panda and C. Providencia, Phys. Rev. C 72, 035802 (2005).

[15] C. Y. Ryu, C. H. Hyun, S. W. Hong and B. T. Kim, Phys. Rev. C 75, 055804 (2007).

[16] P. A. M. Guichon, Phys. Lett. B 200, 235 (1988).

[17] S. Fleck, W. Bentz, K. Shimizu, and Yazaki, Nucl. Phys. A 510, 731 (1990).

[18] X. Jin and B. K. Jennings, Phys. Rev. C 54, 1427 (1996).

[19] K. Tsushima, K. Saito, J. Haidenbaur and A. W. Thomas, Nucl. Phys. A 630, 691 (1998).

[20] K. Tsushima, K. Saito, A. W. Thomas and S. V. Wright, Phys. Lett. B 429, 239 (1998).

[21] C. Y. Ryu, C. H. Hyun, J. Y. Lee and S. W. Hong, Phys. Rev. C 72, 045206 (2005).

[22] S. Pal, M. Hanauske, I.Zakout, H. Stocker and W. Greiner, Phys. Rev. C 60, 015802 (1999). 